JOURNAL OF THEORETICAL

AND APPLIED MECHANICS

54, 2, pp. 645-657, Warsaw 2016

DOI: $10.15632 /$ jtam-pl.54.2.645

\title{
CABLE-SUSPENDED CPR-D TYPE PARALLEL ROBOT
}

\author{
MirJana Filipovic \\ Mihajlo Pupin Institute, University of Belgrade, Belgrade, Serbia; e-mail: mira@robot.imp.bg.ac.rs \\ ANA DJURIC \\ Wayne State University, Detroit, U.S.A; e-mail: ana.djuric2@wayne.edu
}

\begin{abstract}
This paper deals with the analysis and synthesis of a newly selected Cable-suspended Parallel Robot configuration, named CPR-D system. The camera carrier workspace has the shape of a parallelepiped. The CPR-D system has a unique Jacobian matrix that maps the relationship between internal and external coordinates. This geometric relationship is a key solution for the definition of the system kinematic and dynamic models. Because of the CPR-D system complexity, the Lagrange principle of virtual work has been adapted. Two significant Examples have been used for the CPR-D system analysis and validation.
\end{abstract}

Keywords: cable-suspended parallel robot, camera observation, kinematics, dynamics

\section{Introduction}

A system for observation of a workspace with moving objects has been developed to some extent and widely analyzed in various research areas as well as for different applications. Similar systems have been analyzed and modeled as presented by numerous publications.

The kinematic design of a planar three-degree-of-freedom parallel manipulator was considered by Gosselin and Grenier (2011). Four optimal different design criteria were established and analyzed. A trajectory planning approach for cable-suspended parallel mechanisms was presented by Gosselin et al. (2012). A planar two-degree-of-freedom parallel mechanism was used in the analysis. Carricato (2011) studied the kinematics and statics of under-constrained cable-driven parallel robots with less than six cables in crane configuration. A motion controller for a six DOF tendon-based parallel manipulator (driven by seven cables) which moves a platform with high speed was introduced by Fang et al. (2004). A control design of the CPR systems was investigated by Kraus et al. (2013), and Avci et al. (2014). The workspace conditions and the dynamics of the manipulator were described in details. Borgstrom et al. (2007) presented algorithms that enabled precise trajectory control of the Networked Info Mechanical Systems (NIMS), and under constrained three-dimensional (3D) cabled robot intended for use in actuated sensing. Several prototypes of the wire-driven parallel robots with different actuation schemes were presented by Merlet (2010). Two of them were evaluated through extensive tests and showed unexpected kinematic problems. The determination of this workspace was an important issue by Gouttefarde et al. (2006) since the cables can only pull and not push on the mobile platform.

Parallel cable-driven Stewart-Gough platforms consist of an end-effector which is connected to the machine frame by motor driven cables. Since the cables can transmit only tension forces, at least $m=n+1$ cables are needed to tense a system having $n$-degrees-of-freedom. This will cause a kinematical redundancy and leads to an $(m-n)$-dimensional solution space for the cable force distribution presented by Bruckmann et al. (2007). The recent result from a newly designed parallel wire robot which is currently under construction was presented by Pott (2008). It is used for developing a new technique for computation and transfer of its workspace to the available CAD software. An auto-calibration method for over constrained cable-driven parallel 
robots using internal position sensors located in the motors was presented by Miermeister et al. (2012). The wire-driven parallel robot presented by Higuchi et al. (1988) has attracted the interest of researchers since the very beginning of the study of parallel robots. Oh and Agrawal (2005) addressed the issue of control design for a redundant 6-DOF cable robot with positive input constraints. Nonlinear dynamic analysis of the suspended cable system was carried out with some sensible results presented by Duan (1998) that could be useful to the real engineering of LSRT (Large Spherical Radio Telescopes). Integrated mechanical, electronic, optic and automatic control technologies are employed to make considerable improvement upon the same system. A multiple cable robotic crane designed by Shiang et al. (2000) is used to provide improved cargo handling. This is one of a few papers dealing with flexible ropes. For the requirement of trajectory tracking of the LSRT, a large fine tuning platform based on the Stewart platform was presented by Su and Duan (2000a,b). The mathematical model for kinematic control was developed with coordinate transformation, and dynamic analysis was made using a Jacobian matrix with singularity analysis, which built a solid base for the tracking control. Kozak et al. (2006) in their paper addressed the static analysis of cable-driven robotic manipulators with a non-negligible cable mass. A cable suspended parallel robot was analyzed by Zi et al. (2008), in which cables were utilized to replace links to manipulate objects. It was developed from a parallel and serial cable-driven robot. Therefore, a cable system with $j$ end-effectors DOFs requires at least $(j+1)$ cables as shown by Hiller and Fang (2005). For three-translational motions of the feed in the system, a four-cable-driven parallel manipulator was developed. The goal of Yao et al. (2010) was to optimize dimensions of the four-cable-driven parallel manipulator to meet the workspace requirement of the constraint condition in terms of cable tension and stiffness.

However, the same CPR can be used in many different applications, but only some configurations can be used for the workspace observation.

In this paper, we present a novel construction of the CPR system which is named CPR-D, see Figs. 1 and 2. The camera carrier hangs over ropes properly connected to four highest points, i.e. four upper angles of the parallelepiped workspace. The system has been designed with only three motors and two ropes for the maximum workspace, which means that the system is not redundant. This workspace is double bigger than the workspace of similar systems with the same number of motors. The CPR-D system has more advantages in comparison with other similar systems. This system is secured from falling during motion because it is constructed with two parallel ropes, see Fig. 1.

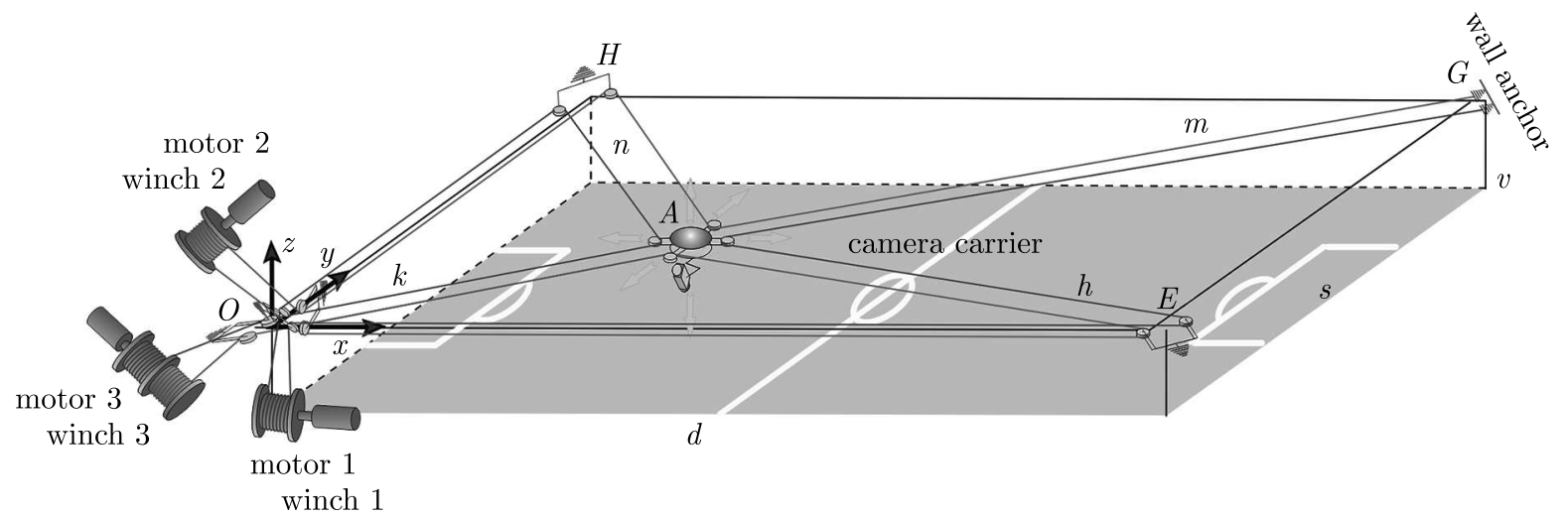

Fig. 1. CPR-D in 3D space

A camera workspace is an area where a camera can move silently and continuously following the observed object. A camera carrier moves freely in the space enabling the shooting of the objects from the above. This gives a unique feeling to the event observer to watch objects from the unusual proximity without disturbances. The observer will be very close to the action 


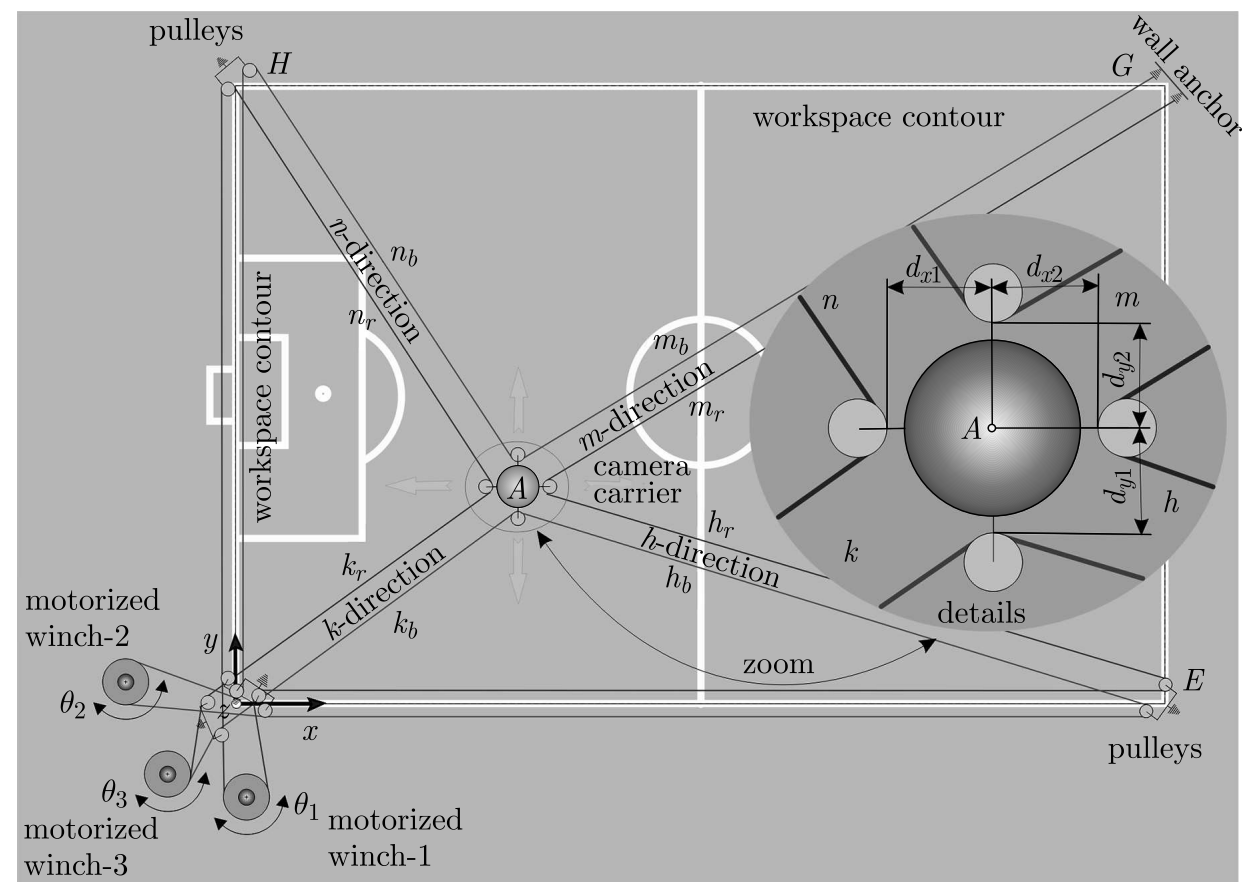

Fig. 2. CPR-D, top view

regardless of the size of the observed space. Motion of the ropes which carry the camera is controlled. The ropes can uncoil or coil, which allow the camera to reach any position in the space. The control system provides three-dimensional motion of the camera. The commands for synchronized motion of each winch are provided by controlling the motion of each motor which ultimately provides the three-dimensional continuous camera carrier motion. The gyroscopic sensor that is installed in the camera carrier is stabilized to the horizon. The nature of this system requires development of a new methodology for calculation of its kinematic and dynamic models, which will be used for building the system.

This work will be extended by implementing elastic properties of the ropes in the kinematic and dynamic models. The research of elasticity dynamics for nonlinear systems was done by the following authors: Raskovic (1965), Rega (2004a,b), Hedrih (2010, 2012). In this paper, the CPR model has been generated using the following assumptions: transverse vibrations of the ropes are neglected and the ropes are unstretchable.

In Section 2, a detailed description of a selected CPR system type and its mathematical model is given. Most of that Section is devoted to its kinematic model, which is directly involved in the development of its dynamic model. Two cases of the system responses are analyzed for different conditions in Section 3, while in Section 4 concluding remarks are presented.

\section{Mathematical model of CPR-D system}

In this research, one subsystem of the CPR family has been selected and analyzed in depth. A graphical representation of that system, named CPR-D, is shown in Figs. 1 and 2. The camera carrier of the CPR-D structure is guided through the work area of the parallelepiped shape with two ropes connected with three winches, each powered by a motor. The ropes coil or uncoil on the winches of radius $R_{1}, R_{2}$ and $R_{3}$. The motors rotate the winches directly, and the motor shafts angular positions after gear boxes are $\theta_{1}, \theta_{2}, \theta_{3}$. This motion moves the camera in the $x$, $y, z$ Cartesian coordinates. 
The first step towards the dynamical model of the CPR-D is the development of its kinematic model. The geometrical relationship between the lengths $k, h, m, n$ and Cartesian coordinates $x, y, z$ is defined by the following equations

$$
\begin{array}{ll}
k=\sqrt{x^{2}+y^{2}+z^{2}} & h=\sqrt{(d-x)^{2}+y^{2}+z^{2}} \\
m=\sqrt{(d-x)^{2}+(s-y)^{2}+z^{2}} & n=\sqrt{x^{2}+(s-y)^{2}+z^{2}}
\end{array}
$$

In Fig. 3, motions of motors 1 and 2 are depicted. Motor 1 (motor 2 as well) works so that it wounds its corresponding rope from one side and unwounds from other side.

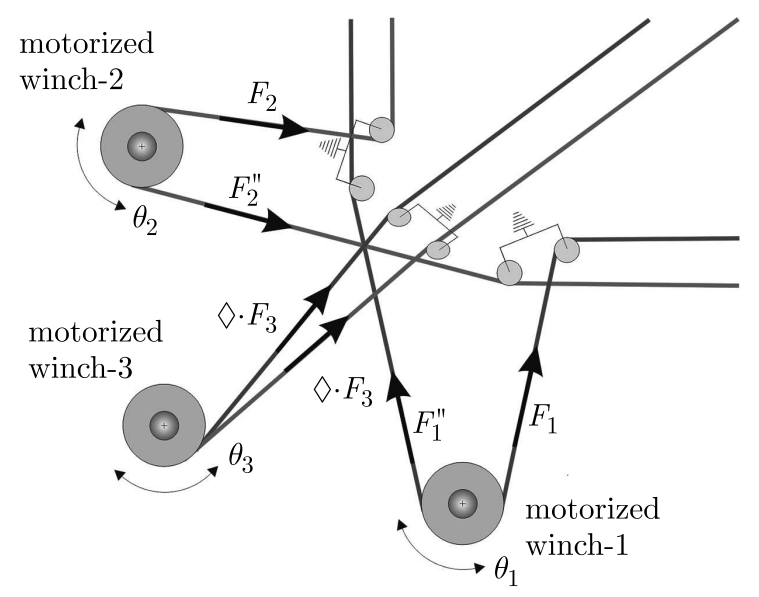

Fig. 3. Rope forces before motor 1, 2 and 3 and after motor 1 and 2

Motions of motors 1 and 2 toward the wall anchors (we call this line "before" motor) are expressed with the following equations respectively

$$
\frac{\Delta \theta_{1}}{\Delta t} R_{1}=\frac{\Delta h}{\Delta t}+\frac{\Delta m}{\Delta t} \quad \frac{\Delta \theta_{2}}{\Delta t} R_{2}=\frac{\Delta m}{\Delta t}+\frac{\Delta n}{\Delta t}
$$

The third motor is used to wind up the two ropes about coil 3 in the $k, h, m, n$ directions. This motion produces winding or unwinding of both ropes at the same time. This can be seen in Figs. 2 and 3. The winch used for winding the ropes has radius $R_{i}, i=1,2,3$. The relation between the third motor motion changes $\Delta \theta_{3}$, and the lengths change $\Delta k, \Delta h, \Delta m, \Delta n$ can be expressed either with equation $(2.3)_{1}$ or $(2.3)_{2}$

$$
\frac{\Delta \theta_{3}}{\Delta t} R_{3}=\frac{\Delta k}{\Delta t}+\frac{\Delta h}{\Delta t}+\frac{\Delta \theta_{2}}{\Delta t} R_{2} \quad \frac{\Delta \theta_{3}}{\Delta t} R_{3}=\frac{\Delta k}{\Delta t}+\frac{\Delta n}{\Delta t}+\frac{\Delta \theta_{1}}{\Delta t} R_{1}
$$

Equation (2.4) is obtained by substituting equation $(2.2)_{2}$ into $(2.3)_{1}$, or equation $(2.2)_{1}$ into $(2.3)_{2}$

$$
\frac{\Delta \theta_{3}}{\Delta t} R_{3}=\frac{\Delta k}{\Delta t}+\frac{\Delta h}{\Delta t}+\frac{\Delta m}{\Delta t}+\frac{\Delta n}{\Delta t}
$$

If the sampling time $\Delta t$ is small enough then equations (2.2) and (2.4) can be expressed, respectively, as

$$
\dot{\theta}_{1} R_{1}=\dot{h}+\dot{m} \quad \dot{\theta}_{2} R_{2}=\dot{m}+\dot{n} \quad \dot{\theta}_{3} R_{3}=\dot{k}+\dot{h}+\dot{m}+\dot{n}
$$

By differentiating equations (2.1) and substituting them into equations (2.5), the relationship between the velocities of the camera carrier in the Cartesian space $\dot{\mathbf{p}}=[\dot{x}, \dot{y}, \dot{z}]^{\mathrm{T}}$ and the velocities of the internal coordinates $\dot{\phi}=\left[\dot{\theta}_{1}, \dot{\theta}_{2}, \dot{\theta}_{3}\right]^{\mathrm{T}}$ can been obtained as following

$$
\dot{\phi}=\mathbf{J}_{d} \dot{\mathbf{p}}
$$


This procedure is named KinCPRD-Solver (Kinematic Cable Parallel Robot D-type Solver). It is clear that the Jacobian matrix $\mathbf{J}_{d}[1 / \mathrm{m}]$ is a full matrix, and its elements beyond the diagonal show strong coupling between the external and internal coordinates.

The kinetic energy $E_{k}$ and the potential energy $E_{p}$ of the camera carrier motion with mass $m_{c}$ are given in the following equations

$$
E_{k}=\frac{1}{2} m_{c} \dot{x}^{2}+\frac{1}{2} m_{c} \dot{y}^{2}+\frac{1}{2} m_{c} \dot{z}^{2} \quad E_{p}=m_{c} g z
$$

The gravitational acceleration is $g=9.81 \mathrm{~m} / \mathrm{s}^{2}$. The CPR-D system has three motors, and their mathematical model is expressed with vector equation (2.8) (Vukobratovic, 1989)

$$
\mathbf{u}=\mathbf{G}_{v} \ddot{\boldsymbol{\phi}}+\mathbf{L}_{v} \dot{\phi}+\mathbf{S}_{v} \mathbf{M}_{d}
$$

where $\mathbf{u}=\left[u_{1}, u_{2}, u_{3}\right]^{\mathrm{T}}$ is voltage, $\mathbf{G}_{v}=\operatorname{diag}\left(G_{v i}\right)$ - motor inertia characteristic, $\mathbf{L}_{v}=$ $\operatorname{diag}\left(L_{v i}\right)$ - motor damping characteristic, $\mathbf{S}_{v}=\operatorname{diag}\left(S_{v i}\right)$ - motor geometric characteristic, $\mathbf{M}_{d}$ - motor load torque

$$
G_{v i}=\frac{J_{r i} R_{r i}}{C_{M i}} \quad L_{v i}=\frac{R_{r i} B_{C i}}{C_{M i}}+C_{E i} \quad S_{v i}=\frac{R_{r i}}{C_{M i}}
$$

where: $R_{r i}[\Omega]$ is rotor circuit resistance, $C_{E i}[\mathrm{~V} /(\mathrm{rad} / \mathrm{s})]$ - back electromotive force constant, $C_{M i}[\mathrm{Nm} / \mathrm{A}]$ - constant of torque proportionality, $B_{C i}[\mathrm{Nm} /(\mathrm{rad} / \mathrm{s})]-$ coefficient of viscous friction, $J_{r i}\left[\mathrm{~kg} \mathrm{~m}^{2}\right]-$ moment of inertia for the rotor and the gear box.

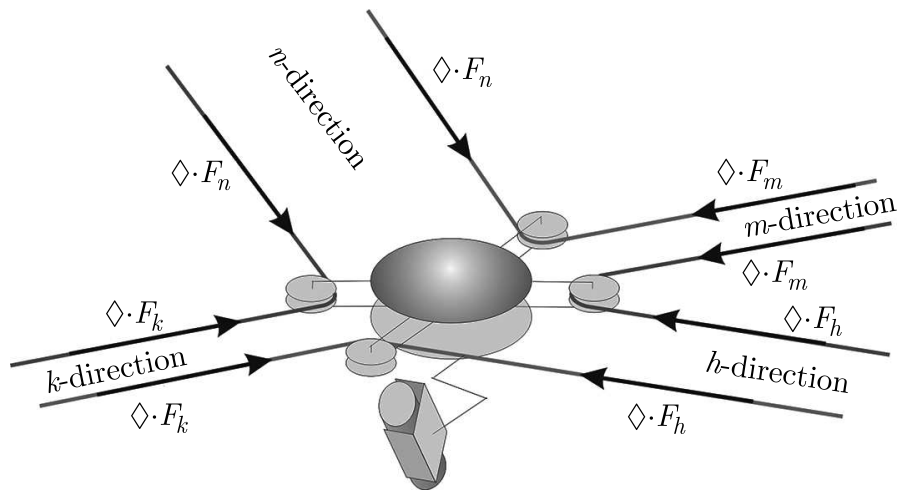

Fig. 4. Rope forces carrying the camera

Vector equation (2.8) is based on Lagrange's equation of motion. The angular positions of the motors shafts $\theta_{1}, \theta_{2}, \theta_{3}$ are selected as generalized coordinates.

The motor load torque $\mathbf{M}_{d}$ is defined with vector equation

$$
\mathbf{M}_{d}=\left[F_{1} R_{1}, F_{2} R_{2}, F_{3} R_{3}\right]^{\mathrm{T}}
$$

The load force $\mathbf{F}_{d}$ includes three components expressed in a vector form as $\mathbf{F}_{d}=\left[F_{1}, F_{2}, F_{3}\right]^{\mathrm{T}}$. This force is acting on the shaft of each motor, and its value depends on the external force $\mathbf{F}$. The external force $\mathbf{F}=\left[F_{x}, F_{y}, F_{z}\right]^{\mathrm{T}}$ represents the sum of the camera inertial force $\mathbf{F}_{p}$ which is acting on the camera carrier described in equation $(2.11)_{2}$ and the perturbation force $\mathbf{P}_{p}$ which is disturbing the camera motion

$$
\mathbf{F}=\mathbf{F}_{p}+\mathbf{P}_{p} \quad \mathbf{F}_{p}=m\left(\ddot{\mathbf{p}}+\mathbf{a}_{c c}\right)
$$

The vector $\mathbf{a}_{c c}=[0,0,-g]^{\mathrm{T}}$ represents the gravitational acceleration. The next step is to describe the dynamic balance between the force $\mathbf{F}_{m}$ in the $m$ rope direction and the external force $\mathbf{F}$, see 


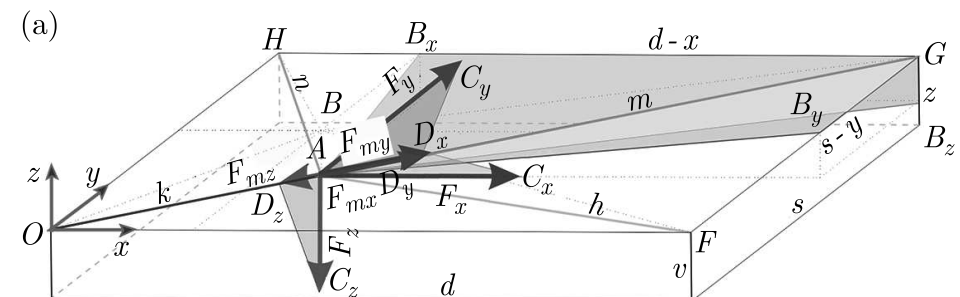

(c)

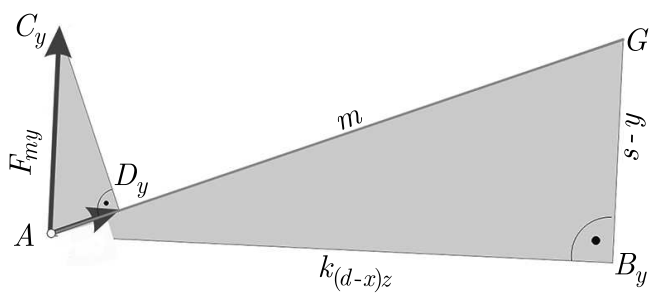

(b)

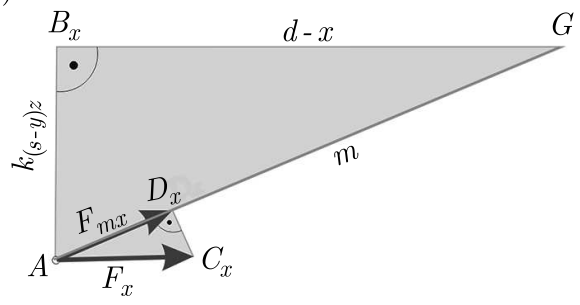

(d)

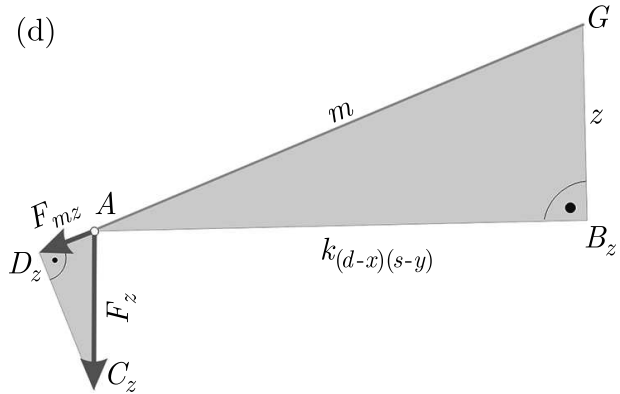

Fig. 5. (a) Characteristic triangles in 3D space, (b) characteristic triangle in the $(d-x)-m$ plane,

(c) characteristic triangle in the $(s-y)-m$ plane, (d) characteristic triangle in the $z-m$ plane

Figs. 5a-5d. Two similar right-angle triangles in the $(d-x)-m$ plane, in the $(s-y)-m$ plane, and in the $z-m$ plane are presented in $2 \mathrm{D}$ space, see Figs. 5b-5d, respectively. All of these triangles can be seen in $3 \mathrm{D}$ space, which is shown in Fig. 5a. The hypotenuses of the $G A B_{x}, G A B_{y}$, $G A B_{z}$ triangles has length $m$, which is changeable during motion of the camera. The other two sides of the $G A B_{x}, G A B_{y}, G A B_{z}$ triangles have sizes $d-x$ and $k_{(s-y) z}=\sqrt{(s-y)^{2}+z^{2}}$, $s-y$ and $k_{(d-x) z}=\sqrt{(d-x)^{2}+z^{2}}, z$ and $k_{(d-x)(s-y)}=\sqrt{(d-x)^{2}+(s-y)^{2}}$, respectively. The component of the external force $\mathbf{F}$ in the $x$ direction is $F_{x}$, in the $y$ direction is $F_{y}$, in the $z$ direction is $F_{z}$. The projection forces $F_{x}, F_{y}, F_{z}$ on the $m$ direction are $F_{m x}, F_{m y}, F_{m z}$, respectively, which can be seen in Figs. 5b-5d. The similarities of the two triangles in Fig. 5 produce the following relations

$$
\begin{array}{lll}
\frac{d-x}{m}=\frac{F_{m x}}{F_{x}} & F_{m x}=\frac{d-x}{m} F_{x} & \frac{s-y}{m}=\frac{F_{m y}}{F_{y}} \\
F_{m y}=\frac{s-y}{m} F_{y} & \frac{z}{m}=\frac{F_{m z}}{F_{z}} & F_{m z}=\frac{z}{m} F_{z}
\end{array}
$$

The force $F_{m}$ is a sum of the previously defined components and it is expressed in the following equation

$$
F_{m}=F_{m x}+F_{m y}+F_{m z}=\frac{d-x}{m} F_{x}+\frac{s-y}{m} F_{y}+\frac{z}{m} F_{z}
$$

The Lagrange principle of virtual work has been used to find the relation between the motor load torque $\mathbf{M}_{d}$ and the external force $\mathbf{F}$

$$
\left(\mathbf{M}_{d}\right)^{\mathrm{T}} \dot{\boldsymbol{\phi}}=\mathbf{F}^{\mathrm{T}} \dot{\mathbf{p}}
$$

By substituting equations (2.6) into (2.14), the following equations are generated

$$
\left(\mathbf{M}_{d}\right)^{\mathrm{T}} \mathbf{J}_{d} \dot{\mathbf{p}}=\mathbf{F}^{\mathrm{T}} \dot{\mathbf{p}} \quad\left(\mathbf{J}_{d}\right)^{\mathrm{T}} \mathbf{M}_{d}=\mathbf{F}
$$

From $(2.15)_{2}$, equation (2.16) can be expressed as

$$
\mathbf{M}_{d}=\left(\left(\mathbf{J}_{d}\right)^{\mathrm{T}}\right)^{-1} \mathbf{F}
$$

Equation (2.16) cannot be directly applied to the system from Figs. 1 and 2 because of the following reasons: 
- The system has two ropes in each direction. Equation (2.16) has been corrected using the factor $\diamond=0.5$.

- Motor 3 is used to synchronize winding or unwinding of the two ropes.

These will produce the adopted Jacobian matrix $J_{x \diamond d}$. The matrix $\mathbf{O}_{d}[\mathrm{~m}]$ is generated, which represents the torque mapping matrix, as defined below

$$
\mathbf{O}_{d}=\left(\left(J_{x \diamond d}\right)^{\mathrm{T}}\right)^{-1}
$$

The adapted Lagrange's principle of virtual work has been used for solving the complex relation between the motor load torque $\mathbf{M}_{d}$ (acting as a load on the first, second and third motor shaft) and external forces $\mathbf{F}$ (acting on the camera carrier)

$$
\mathbf{M}_{d}=\mathbf{O}_{d} \mathbf{F} \quad \mathbf{u}=\mathbf{G}_{v} \ddot{\boldsymbol{\phi}}+\mathbf{L}_{v} \dot{\boldsymbol{\phi}}+\mathbf{S}_{v} \mathbf{O}_{d} \mathbf{F}
$$

The torque mapping matrix $\mathbf{O}_{d}[\mathrm{~m}]$ indicates that the system is highly coupled. The control law is selected by the local feedback loop for the position and velocity of the motor shaft in the following form

$$
u_{i}=K_{l p i}\left(\theta_{i}^{o}-\theta_{i}\right)+K_{l v i}\left(\dot{\theta}_{i}^{o}-\dot{\theta}_{i}\right)
$$

where $K_{l p i}$ is a position constant, and $K_{l v i}$ is a velocity constant for the motion control.

The comparison between previously published papers and this research is summarized as in the following:

- The novel KinCPRD-Solver gives a relation between the internal and external coordinates through the unique Jacobian matrix $\mathbf{J}_{d}$.

- All three motors in this system are differently integrated in comparison with the previously published systems.

- Most of the previously published papers do not involve dynamics of the motor. The CPR-D system includes the motors which significantly influence the total system response.

- The CPR-D system construction requires a novel dynamic relation between the load forces which are acting on the camera and the forces in the $m$ direction.

- The previously published systems used Lagrange's principle of virtual work for calculating the relation between the external and internal forces in the original form. This system has double ropes in all four directions, which requires an adaptation of the Lagrange principle of virtual work.

\section{Simulation results}

The CPR-D system presented in Figs. 1 and 2 is modeled and analyzed by the software package AIRCAMD. The software package AIRCAMD is used for validation of applied theoretical contributions. This software includes three essential modules which are kinematic, dynamic and motion control law solvers for the CPR-D system. The most important element of the CPR-D system is the mathematical model of the motor which is an integral part of the software package AIRCAMD. Through the simulation results, it is shown that the dynamic characteristics of the motor significantly affect the response of the system and its stability. In order to make the results comparable, simulation is made for the same desired system parameters. The camera carrier motion dynamics directly depends on the mechanism dynamic parameters. The camera moves in the 3D space ( $x, y, z$ directions). The workspace is characterized by length $d=3.2 \mathrm{~m}$, width $s=2.2 \mathrm{~m}$ and height $v=2.0 \mathrm{~m}$ of the recorded field. The position of the camera carrier 
in the Cartesian space is $\mathbf{p}=[x, y, z][\mathrm{m}]$. The starting point is $\mathbf{p}_{\text {start }}^{o}=[0.3 \mathrm{~m}, 1.8 \mathrm{~m},-0.2 \mathrm{~m}]$, and the end point $\mathbf{p}_{\text {end }}^{o}=[0.9 \mathrm{~m}, 1.2 \mathrm{~m},-0.9 \mathrm{~m}]$, They are presented in Fig. 6a, while their reference velocities are shown in Fig. 6c. In Fig. 6b, we show the reference composite velocity of the camera carrier. The shape of the composite velocity is trapezoidal.
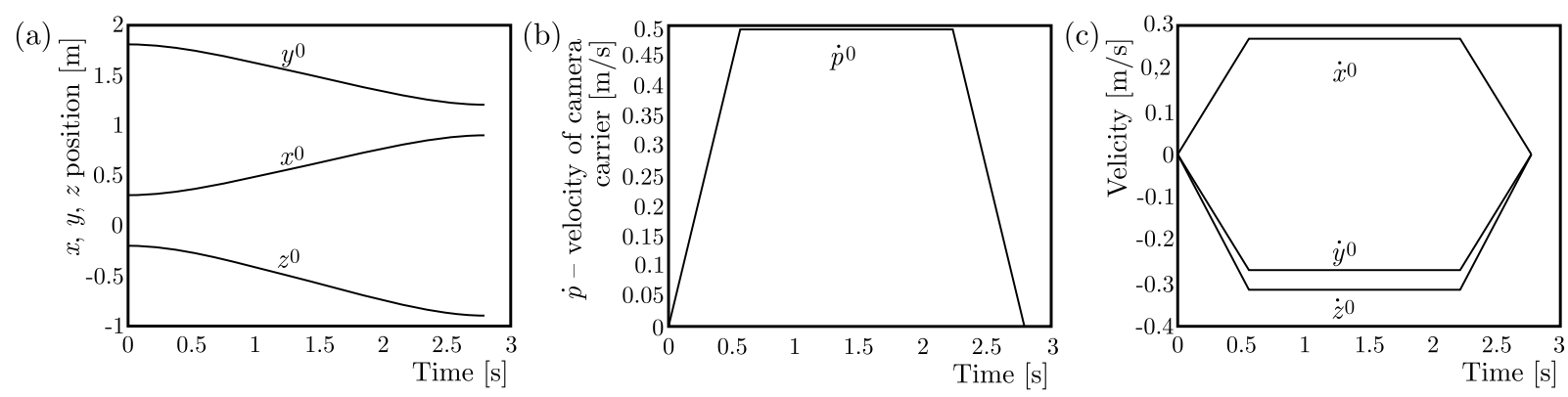

Fig. 6. The reference trajectory motion of the camera carrier (a) position $x^{o}, y^{o}, z^{o}$, (b) velocity (maximum value: $p_{\max }^{o}=0.494 \mathrm{~m} / \mathrm{s},(\mathrm{c})$ velocity components $\dot{x}^{o}, \dot{y}^{o}, \dot{z}^{o}($ Examples 1,2$)$

The motors are Heinzman SL100F type and gears are HFUC14-50-2A-GR+belt type. The characteristics of the motors are: $R_{r i}=0.917 \Omega$ - rotor circuit resistance, $C_{E i}=3.3942 \mathrm{~V} /(\mathrm{rad} / \mathrm{s})$ - back electromotive force constant, $C_{M i}=2.5194 \mathrm{Nm} / \mathrm{A}$ - constant of torque proportionality, $B_{C i}=0.0670 \mathrm{Nm} /(\mathrm{rad} / \mathrm{s})$ - coefficient of viscous friction, $J_{r i}=1.5859 \mathrm{~kg} \mathrm{~m}^{2}$ - moment of inertia for the rotor and the gear box.

The sample time is $d t=0.0001 \mathrm{~s}$. The positional and velocity motion controller parameters are $K_{l p i}=4200$ and $K_{l v i}=130$, respectively. Winches radii are $R_{i}=0.15 \mathrm{~m}$. The system responses are comparable and therefore are shown in Table 1. The results for Example 1 are presented in Figs. 7 and 10a, while for Example 2 are presented in Figs. 8 and 10b. Figure 7 (and Fig. 8 as well) has six pictures related to:

a) camera carrier position at the reference and the real frames,

b) motor shaft position at the reference and the real frames,

c) load force at the reference and the real frames,

d) deviation between the real and the reference trajectory of the camera carrier,

e) deviation between the real and the reference trajectory of the motor shaft positions,

f) control signals at the reference and the real frames.

Table 1. Comparison of two selected Examples

\begin{tabular}{|l|c|c|}
\hline Example & 1 & 2 \\
\hline Figure & $7,10 \mathrm{a}$ & $8,10 \mathrm{~b}$ \\
\hline $\begin{array}{l}\text { Mathematical model of the system at the reference frame } \\
\text { is defined by equations }\end{array}$ & $(2.1)-(2.19)$ & $(3.1)-(3.5)$ \\
\hline Camera carrier is under the influence of the disturbance force & yes & yes \\
\hline System at the reference frame is coupled & yes & no \\
\hline
\end{tabular}

The CPR-D system is designed for outdoor use. Because of that, we analyzed the system behavior under the influence of wind impacts, determined as the force $\mathbf{F}_{p}=[100(\sin (4 \pi t)+$ $\sin (32 \pi t)), 0,0]^{\mathrm{T}}$, see Fig. 9. The force has a sine shape and operates only in the $x$ direction, while the components in the $y$ and $z$ directions are zero.

Example 1: The motion response of the camera carrier has oscillatory characteristics and angular positions of all three motors are caused by the sinusoidal disturbance force. There is a very good 
(a)
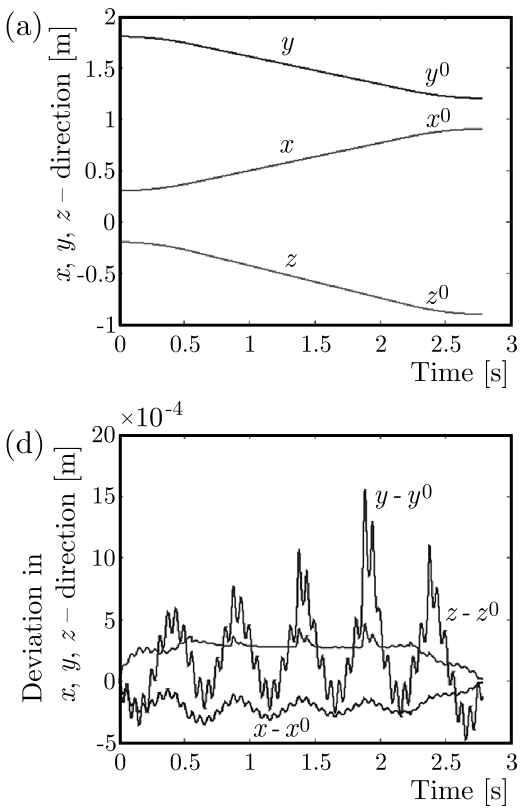

(a)
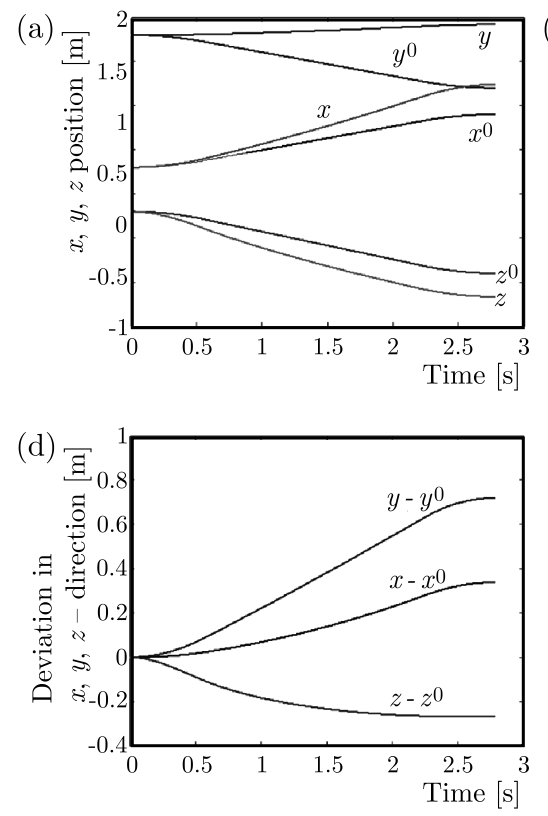
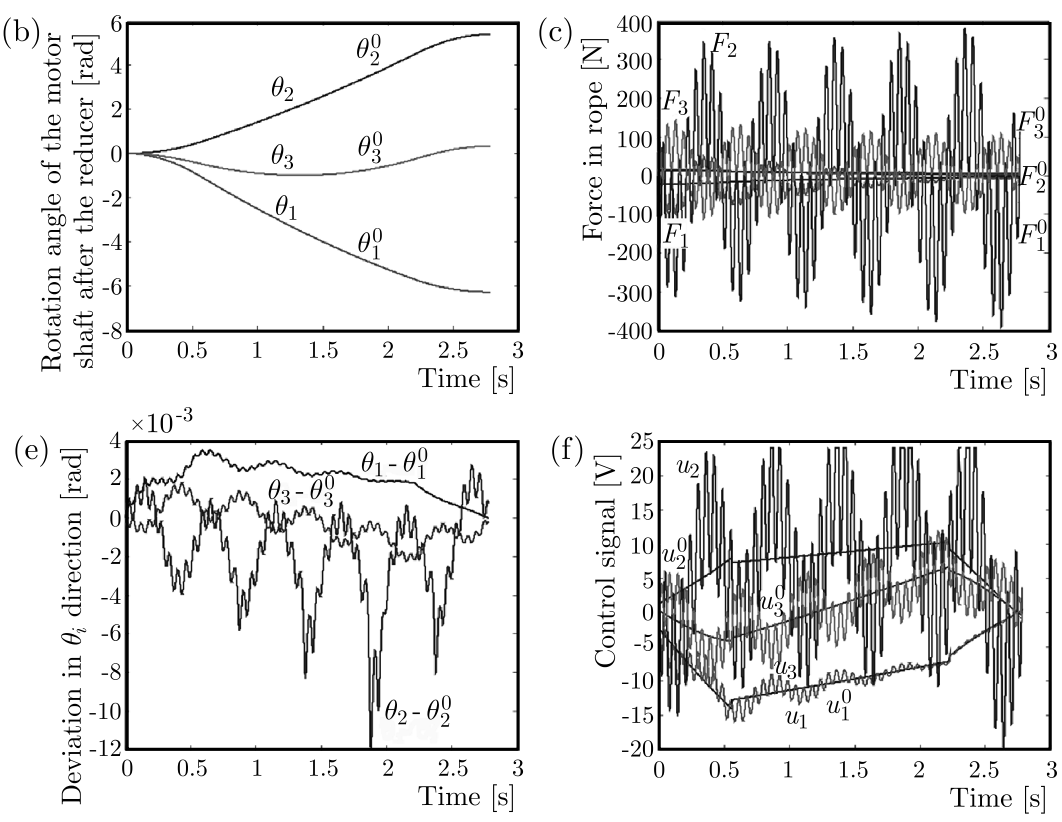

Fig. 7. Example 1
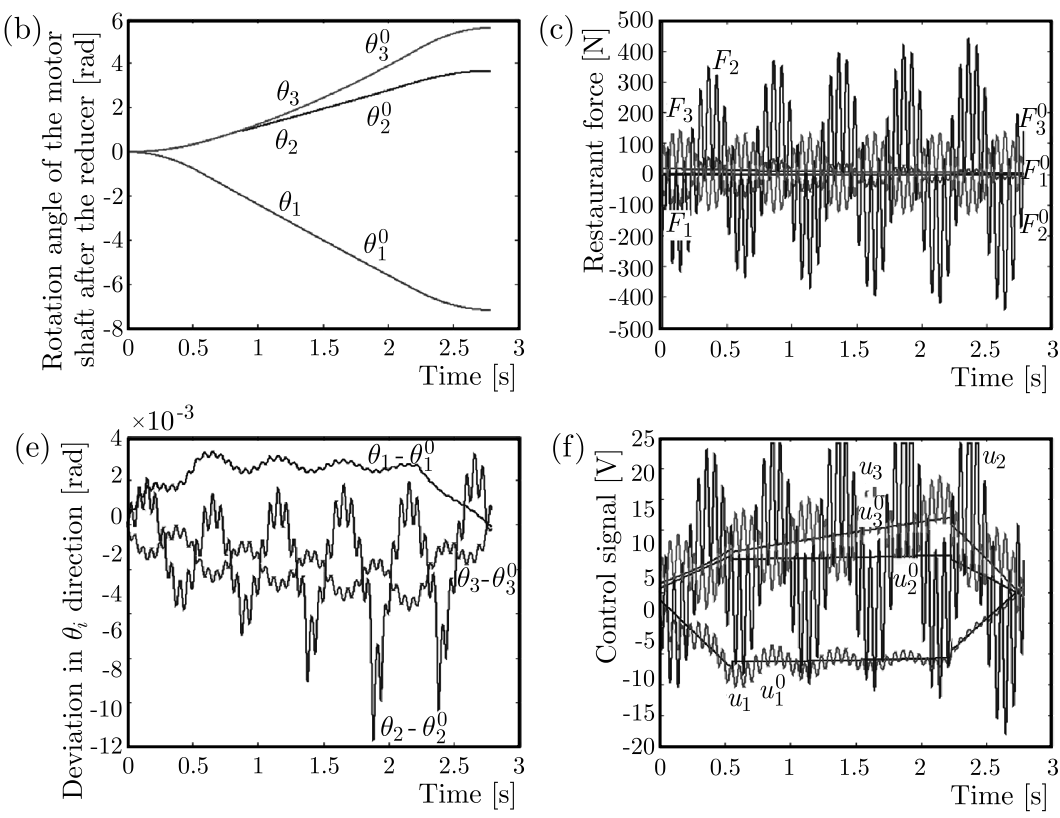

Fig. 8. Example 2

tracking of the desired trajectory at the camera carrier real frame and at the motor motion real frame, until the moment when motor 2 enters the saturation in oscillatory manner, see Fig. 7f. The first and the third motor do not enter the saturation at all. The force $F_{m}$ has two components of the same magnitude $F_{m 1}=F_{m 2}$ acting in each rope in the $m$ direction at the reference and the real frames, which is presented in Fig. 10a.

Example 2: All system and control parameters are the same as in Example 1. This Example is done with one illogical assumption, which is that the system at the reference frame is uncoupled. In that case, the Jacobian matrix $\mathbf{J}_{d \oplus}$ has the diagonal form

$$
\dot{\boldsymbol{\phi}}=\mathbf{J}_{d \oplus \dot{\mathbf{p}}}
$$




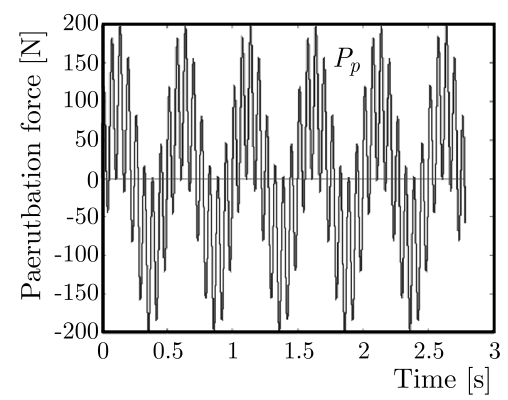

Fig. 9. Perturbation force at the camera carrier (Examples 1,2)
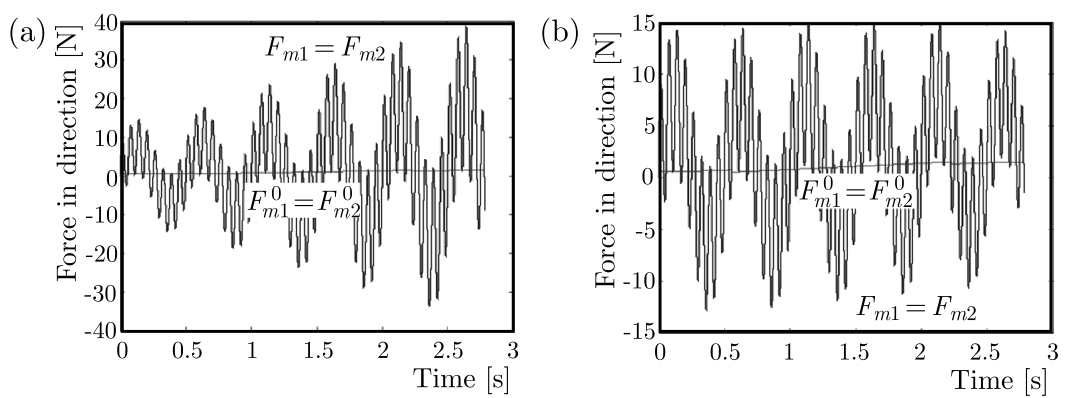

Fig. 10. Components of the force $F_{m}$ acting in the $m$ direction at the reference and the real frames, (a) Example 1, (b) Example 2

The mathematical model of the system has the following form

$$
\mathbf{u}=\mathbf{G}_{v} \ddot{\boldsymbol{\phi}}+\mathbf{L}_{v} \dot{\boldsymbol{\phi}}+\mathbf{S}_{v} \mathbf{M}_{d \oplus}
$$

Using the adapted Lagrange principle of virtual work, the relationship between the motor load torque $\mathbf{M}_{d \oplus}$ and the external force $\mathbf{F}$ has been given below

$$
\mathbf{M}_{d \oplus}=\mathbf{O}_{d \oplus} \mathbf{F}
$$

The diagonal adopted Jacobian matrix is $\mathbf{J}_{x \diamond d \oplus}$. The matrix $\mathbf{O}_{d \oplus}$ is generated, which represents the torque mapping matrix, as defined below

$$
\mathbf{O}_{d \oplus}=\left(\left(\mathbf{J}_{x \diamond d \oplus}\right)^{\mathrm{T}}\right)^{-1}
$$

The torque mapping matrix $\mathbf{O}_{d \oplus}$ is diagonal like the Jacobian matrix $\mathbf{J}_{d \oplus \text {. Substituting (3.3) }}$ into equation (3.2) produces a dynamic model of the uncoupled CPR-D system at the reference frame

$$
\mathbf{u}=\mathbf{G}_{v} \ddot{\boldsymbol{\phi}}+\mathbf{L}_{v} \dot{\boldsymbol{\phi}}+\mathbf{S}_{v} \mathbf{O}_{d \oplus} \mathbf{F}
$$

In this case, the mathematical model of the CPR-D system at the reference frame is defined by equations (3.1)-(3.5). At the real frame, the system is coupled and its kinematic and dynamic models are defined by equations (2.1)-(2.19), see Fig. 8. The coupling characteristics are not taken into the consideration at the reference frame. Due to that fact, the tracking of the referent trajectory in the Cartesian space is not satisfactory, see Figs. 8a and 8d.

The position control law produces the ideal response of the motor angular motion, see Figs. 8b and $8 \mathrm{e}$.

The forces $F_{m 1}=F_{m 2}$ acting in each rope in the $m$ direction at the reference and real frames are presented in Fig. 10b. Example 2 has an important theoretical meaning, because it confirms the strong coupling between the external and internal coordinates. 
The presented results imply that the dynamics of the individual motor significantly depends of the selection of the CPR-D structure and its parameters. The CPR-D is modeled and analyzed by the software package AIRCAMD.

\section{Conclusion}

The highly authentic general mathematical model for the CPR-D system has been developed. This model represents novel kinematic and dynamic solutions of the complex Cable suspended Parallel Robot structure. The CPR-D system is selected to carry the camera through four pivot points which produce a 3D workspace of a parallelepiped shape. The camera carrier is controlled by two ropes in each of the three directions, and driven by three motors. The kinematic model is defined for the monitored system via the Jacobian matrix. The generalized coordinates selected for the CPR-D model are angular positions of the motors $\theta_{1}, \theta_{2}, \theta_{3}$ named internal coordinates. Camera motion is defined in the Cartesian space, described with the $x, y, z$ coordinates, named the external coordinate system. The relation between the internal and external coordinate systems is described by the Jacobian matrix $\mathbf{J}_{d}$. This relation represents the kinematic model of the CPR-D system. The solution for the CPR-D kinematic structure has been found through a novel procedure named KinCPRD-Solver (Kinematic Cable Parallel Robot D-type Solver) which is developed and validated using two selected Examples. The relation between the motor load torque and the force acting at the camera carrier is described by the Lagrange principle of virtual work. This calculation shows that in this relation, the Jacobian matrix is involved. Because of the construction complexity of this system, the Lagrange principle of virtual work had to be adapted for two reasons. The software package AIRCAMD has been developed and used for individual analysis of the CPR-D model from various aspects such as selecting different workspace dimensions, camera carrier mass, external disturbances, choice of the control law, reference trajectory, avoidance of singularity and many other characteristics.

The future research will involve elastic ropes (type of nonlinear dynamic elasticity as defined by Filipovic et al. (2007), Filipovic and Vukobratovic (2008a,b), Filipovic (2012) in the mathematical model of the CPR system. Different CPR models previously developed will be unified according to their similarities into a single reconfigurable model, using the methodology presented by Djuric et al. (2010, 2012). Stability conditions, sensitivity analysis and singularity analysis of the CPR-D system will be done in the future research.

\section{Acknowledgment}

This research has been supported by the Ministry of Education, Science and Technological Development, Government of the Republic of Serbia through the following two projects: Grant TR-35003 "Ambientally intelligent service robots of anthropomorphic characteristics", by Mihajlo Pupin Institute, University of Belgrade, Serbia, and partially supported by the project SNSF Care-robotics project No. IZ74Z0-137361/1 by Ecole Polytechnique Federale de Lausanne, Switzerland.

We are grateful to Prof. Dr. Katica R. (Stevanovic) Hedrih from the Mathematical Institute, Belgrade for helpful consultations during preparation of this paper.

\section{References}

1. Avei E., Kenmochi M., Kawanishi M., Narikiyo T., Kawakami S., Saitoh Y., 2014, Vibration Control of $3 \mathrm{P}(\mathrm{S}) 4$ class parallel mechanisms for high speed applications using quantitative feedback design, IROS 2014 - IEEE/RSJ International Conference on Intelligent Robots and Systems, Chicago, IL, USA

2. Borgstrom P.H., Borgstrom N.P., Stealey M.J., Jordan B., Sukhatme G., Batalin M.A., KAISER W.J., 2007, Discrete trajectory control algorithms for NIMS3D, an autonomous 
underconstrained three-dimensional cabled robot, Proceedings of the 2007 IEEE/RSJ International Conference on Intelligent Robots and Systems, San Diego, CA, USA

3. Bruckmann T., Mikelsons L., Schramm D., Hiller M., 2007, Continuous workspace analysis for parallel cable-driven Stewart-Gough platforms, Special Issue: Sixth International Congress on Industrial Applied Mathematics (ICIAM07) and GAMM Annual Meeting, Zürich, 7, 1

4. Carricato M., 2011, Under-constrained cable-driven parallel robots, [In:] Quarta giornata di studio Ettore Funaioli, 16 luglio 2010, Asterisco, 443-454

5. Djuric A.M., Saidi R.Al, ElMaraghy W.H., 2010 Global kinematic model generation for n-DOF reconfigurable machinery structure, 6th IEEE Conference on Automation Science and Engineering, CASE 2010, Toronto, Canada

6. Djuric A., Saidi R.Al, ElMaraghy W.H., 2012, Dynamics solution of n-DOF global machinery model, Robotics and Computer Integrated Manufacturing (CIM) Journal, 28, 5, 621-630

7. DuAn B.Y., 1998, A new design project of the line feed structure for large spherical radio telescope and its nonlinear dynamic analysis, Mechatronics, 9, 53-64

8. Fang S., Franitza D., Torlo M., Bekes F., Hiller M., 2004, Motion control of a tendon-based parallel manipulator using optimal tension distribution, IEEE/ASME Transactions of Mechatronics, 9, 3, 561-568

9. Filipovic M., 2012, Relation between Euler-Bernoulli equation and contemporary knowledge in robotics, Robotica, 30, 1-13

10. Filipovic M., Potkonjak V., Vukobratovic M., 2007, Humanoid robotic system with and without elasticity elements walking on an immobile/mobile platform, Journal of Intelligent and Robotic Systems, 48, 157-186

11. Filipovic M., Vukobratovic M., 2008a, Complement of source equation of elastic line, Journal of Intelligent and Robotic Systems, 52, 2, 233-261

12. Filipovic M., Vukobratovic M., 2008b, Expansion of source equation of elastic line, Robotica, 26, 6, 739-751

13. Gosselin C., Grenier M., 2011, On the determination of the force distribution in overconstrained cable-driven parallel mechanisms, Meccanica, 46, 1, 3-15

14. Gosselin C., Ren P., Foucault S., 2012, Dynamic trajectory planning of a two-DOF cable-suspended parallel robot, International Conference on Robotics and Automation RiverCentre, Saint Paul, Minnesota, USA, May 14-18

15. Gouttefarde M., Merlet J.-P., Daney D., 2006, Determination of the wrench-closure workspace of 6-DOF parallel cable-driven mechanisms, Advances in Robot Kinematics, 5, 315-322

16. Hedrih (Stevanovic) K., 2010, Energy analysis in the hybrid system forced regimes, Proceeding of Institute of Mathematics NANU Ukraine, 7, 3, 90-107

17. Hedrin (Stevanovic) K., 2012, Energy and nonlinear dynamics of hybrid system, [In:] Dynamical Systems and Methods, Albert Luo (Edit.), Springer, 1, 29-83

18. Higuchi T., Ming A., Jiang-Yu J., 1988, Application of multi-dimensional wire crane in construction, 5th International Symposium on Robotics in Construction, Tokyo, June, 6/8, 661-668

19. Hiller M., FANG S.Q., 2005, Design, analysis and realization of tendon-base parallel manipulators, Mechanism and Machine Theory, 40, 429-445

20. Kozak K., Zhou Q.J., Wang J., 2006, Static Analysis of Cable-driven manipulators with non-negligible cable mass, IEEE Transaction on Robotics, 22, 2, 425-433

21. Kraus W., Schmidt V., Rajendra P., Pott A., 2013, Load identification and compensation for a Cable-Driven parallel robot, ICRA 2013, IEEE International Conference on Robotics and Automation, Karlsruhe, Germany, 2485-2490 
22. Merlet J.P., 2010, MARIONET, A family of modular wire-driven parallel robots, Advances in Robot Kinematics: Motion in Man and Machine, 1, 53-61

23. Miermeister P., Pott A., Verl A., 2012, Auto-calibration method for overconstrained cable-driven parallel robots, ROBOTIK 2012 - 7th German Conference on Robotics, Munich, Germany

24. Он S.-R., Agrawal S.K.A., 2005, Reference governor-based controller for a cable robot under input constraints, IEEE Transaction on Control Systems Technology, 13, 4, 639-645

25. Pотт A., 2008, Forward kinematics and workspace determination of a wire robot for industrial applications, Advances in Robot Kinematics: Analysis and Design, 7, 451-458

26. Raskovic D., 1965, Theory of Oscillations, Scientific Book, Belgrade, Serbia

27. Rega G., 2004a, Nonlinear vibrations of suspended cables. Part I: Modeling and analysis, Applied Mechanics Reviews, ASME, 57, 6, 443-478

28. RegA G., 2004b, Nonlinear vibrations of suspended cables, Part II: Deterministic phenomena, Applied Mechanics Reviews, ASME, 57, 6, 479-514

29. Shiang W.-J., Cannon D., Gorman J., 2000, Optimal force distribution applied to a robotic crane with flexible cables, Proceedings of the 2000 IEEE International Conference on Robotics and Automation, San Francisco, Ca, 1948-1954

30. Su X.Y., DuAn B.Y., 2000a, The application of the stewart platform in large spherical radio Telescopes, Journal of Robotic Systems, 17, 7, 375-383

31. Su X.Y., DuAn B.Y., 2000b, The mathematical design and kinematics accuracy analysis of a fine tuning stable platform for the large spherical radio telescope, Mechatronics, 10, 819-834

32. Vukobratovic M., 1989, Introduction to Robotics, Springer-Verlag

33. Yao R., Tang X., Wang J., Huang P., 2010, Dimensional optimization design of the four-cable driven parallel manipulator, FAST, IEEE/ASME Transaction on Mechatronics, 15, 6, 932-941

34. Zi B., DuAn B.Y., Du J.L., BAO H., 2008, Dynamic modeling and active control of a cable-suspended parallel robot, Mechatronics, 18, 1, 1-12 